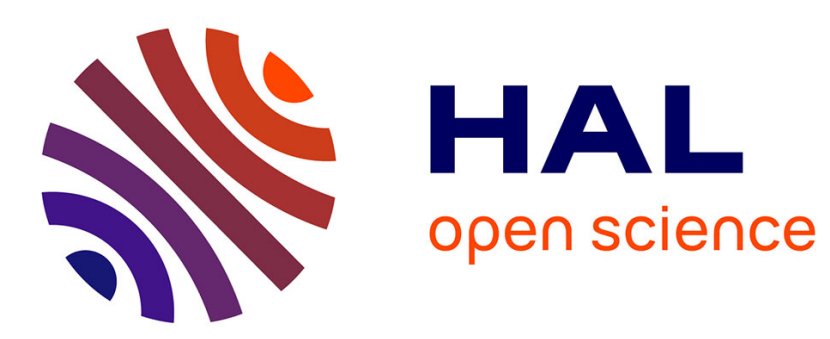

\title{
The PRAE Multidisciplinary Project
}

R. Delorme, D. Marchand, C. Vallerand

\section{- To cite this version:}

R. Delorme, D. Marchand, C. Vallerand. The PRAE Multidisciplinary Project. Nucl.Phys.News, 2019, 29 (1), pp.32-35. 10.1080/10619127.2019.1571833 . hal-02097408

\section{HAL Id: hal-02097408 \\ https://hal.science/hal-02097408}

Submitted on 20 Aug 2020

HAL is a multi-disciplinary open access archive for the deposit and dissemination of scientific research documents, whether they are published or not. The documents may come from teaching and research institutions in France or abroad, or from public or private research centers.
L'archive ouverte pluridisciplinaire HAL, est destinée au dépôt et à la diffusion de documents scientifiques de niveau recherche, publiés ou non, émanant des établissements d'enseignement et de recherche français ou étrangers, des laboratoires publics ou privés. 


\title{
The PRAE Multidisciplinary Project
}

\author{
R. Delorme ${ }^{1}$, D. Marchand ${ }^{2}$, C. Vallerand ${ }^{3}$ \\ ${ }^{1}$ Laboratoire d'Imagerie et Modélisation en Neurobiologie et Cancérologie \\ CNRS-IN2P3, Université Paris-Sud, Université Paris-Saclay et Université Paris Diderot \\ 91405 Orsay cedex, France \\ ${ }^{2}$ Institut de Physique Nucléaire d'Orsay \\ CNRS-IN2P3, Université Paris-Sud, Université Paris-Saclay \\ 91406 Orsay cedex, France \\ ${ }^{3}$ Laboratoire de l'Accélérateur Linéaire \\ CNRS-IN2P3, Université Paris-Sud, Université Paris-Saclay \\ 91898 Orsay cedex, France
}

\section{Introduction}

The PRAE (Platform for Research and Applications with Electrons) facility [1] under construction in the Orsay campus of the Paris-Sud University, is a multidisciplinary platform based on a high-performance new generation linear accelerator delivering a pulsed electron beam in the energy range $30-70 \mathrm{MeV}$, upgradable to $140 \mathrm{MeV}$. This project originates from the complementary expertise of several local scientific research communities involved in radiobiology, subatomic physics, instrumentation and particle accelerator technologies to perform multidisciplinary studies of significant impact. The $50 \mathrm{MeV}$ to $140 \mathrm{MeV}$ electron energy range will be suitable to perform a series of new promising feasibility and radiobiological studies of less destructive radiotherapy treatments relying on space fractionated sub-millimetric pencil beams and other novel techniques. In the field of subatomic physics, we are aiming at a significant contribution to the worldwide effort of understanding the puzzle of the proton charge radius [2]. The ProRad experiment will investigate electron proton elastic scattering to accurately measure the proton electric form factor in an unexplored ultra-low four-momentum squared range. Additionally, a fully equipped instrumentation platform will provide the tools to develop a next generation of detectors used in many research areas such as medical imaging, subatomic and particle physics, spatial technology and astrophysics. With first beam expected by 2021 , the PRAE facility will provide a unique platform available for academic and non-academic communities as well as for the development of outreach programs intended for students.

\section{Accelerator technology}

The PRAE accelerator is a high-performance electron accelerator [3] consisting of a photoinjector, an accelerating section and two beamlines (Fig. 1). A photo-injector using current technology laser will extract the electrons from the photocathode of a radiofrequency (RF) gun. To obtain a high charge per bunch, a metallic magnesium photocathode will be used with a laser pulse energy of a few tens of $\mu \mathrm{J}$ at a wavelength of $260 \mathrm{~nm}$. The RF gun is made of 2.5 copper cells, magnetically coupled to a waveguide. Small emittance is obtained by coupling the photocathode with an accelerating field of $80 \mathrm{MV} / \mathrm{m}$, representing a RF power of $5 \mathrm{MW}$ in a $3 \mu$ s pulse. An instrumented section for beam characterization immediately 
follows. The PRAE acceleration section is a $3.5 \mathrm{~m}$ long High-Gradient (HG) RF S-band (3 GHz) Travelling Wave structure, constituting a compact machine. The final HG structure will provide an energy gain of around $65 \mathrm{MeV}$ for an input peak power of $40 \mathrm{MW}$. The design performance of the PRAE beam at the end of acceleration is summarized in Table 1 . The accelerating section is followed by a $3.5 \mathrm{~m}$ drift, that will host a second accelerating structure in a second phase. The main beamline separates further into direct and deviated lines. The direct line comprises an energy compression system consisting of a magnetic Dtype chicane, involving a movable horizontal collimator, coupled with a dechirping structure for reducing the beam energy spread to the few $10^{-4}$ required for ProRad operation. The deviated line comprises several magnetic elements to cope with the different beams scenarii: electron focusing, mini-beams grid...

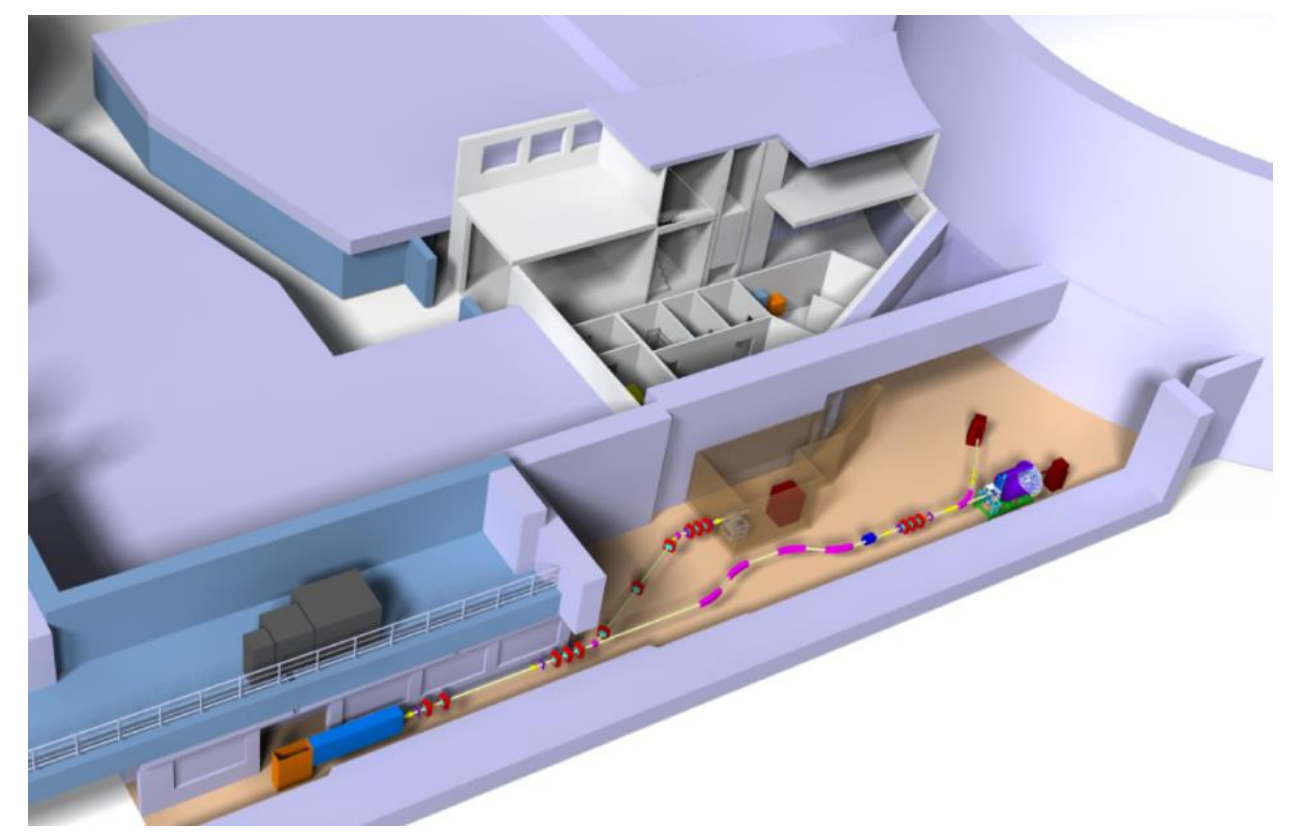

Figure 1: Infrastructure and implantation of the PRAE accelerator.

\begin{tabular}{r|c|r|c} 
Energy $(\mathrm{MeV})$ & $30-70(140)$ & Repetition rate $(\mathrm{Hz})$ & 50 \\
\hline RF frequency $(\mathrm{GHz})$ & 3.0 & Bunch charge $(\mathrm{nC})$ & $0.00005-2$ \\
\hline Normalized emittance $(\mathrm{mm} . \mathrm{mrad})$ & $3-10$ & Bunch number $(/ \mathrm{pulse})$ & 1 \\
\hline Energy spread $(\%)$ & $<0.2$ & Bunch length $(\mathrm{ps})$ & $<10$
\end{tabular}

Table 1: Design performances of the PRAE beam at the end of the accelerating section.

\section{Radiobiology}

Radiation therapy (RT) is one of the major cancer treatment techniques. It aims at delivering a sufficiently high radiation dose to the tumor in order to control the cancer's progression, without causing severe complications to the surrounding healthy organs. However, despite the huge advances in dose-delivery modalities and image-guided RT techniques, some radioresistant cancers cannot be cured, mainly because of the limited dose that can be tolerated by the normal tissues. Most of the RT treatments for superficial tumors are currently delivered using mega-volt photon beams or electron beams with energies smaller than $20 \mathrm{MeV}$. Light ion beam therapy (proton, carbon) is a major breakthrough of recent decades for the improvement of the treatment efficiency, as it allows, among other 
advantages, the complete tissue sparing after the Bragg peak. Unfortunately, the large cost and size of such facilities limit the number of patients that can benefit.

Very high-energy electrons (VHEE: 70-300 MeV) have gained interest for their use in therapy with the improvement of compact accelerator techniques [4], appearing as a potential costeffective alternative between photons and protons. Among their major advantages over photons, one can retain the possibility to scan small pencil beams, producing a finer resolution of intensity modulated treatment, and favorable dosimetric characteristics (low lateral penumbrae and reduced sensitivity to tissue heterogeneities) leading to superior dose distributions for most clinical cases [5]. Another approach to increase the therapeutic window for cancer treatment is to protect the normal tissues by using the concept of Spatially Fractionated Radiotherapy (SFR). In SFR, the resulting dose profiles consist of a pattern of peaks and valleys, with high doses along the beam paths and low doses in the spaces between them. This have been proven safe and effective in delivering large cumulative doses without causing severe damage to healthy tissues, either with the GRID irradiation technique [6] (centimeter-sized collimated beams) or using mini-beams [7] (beam sizes from 400-700 $\mu \mathrm{m}$ ). Small beams allow exploiting the dose-volume effects: the smaller the beam size, the higher the tolerances of the normal tissue.

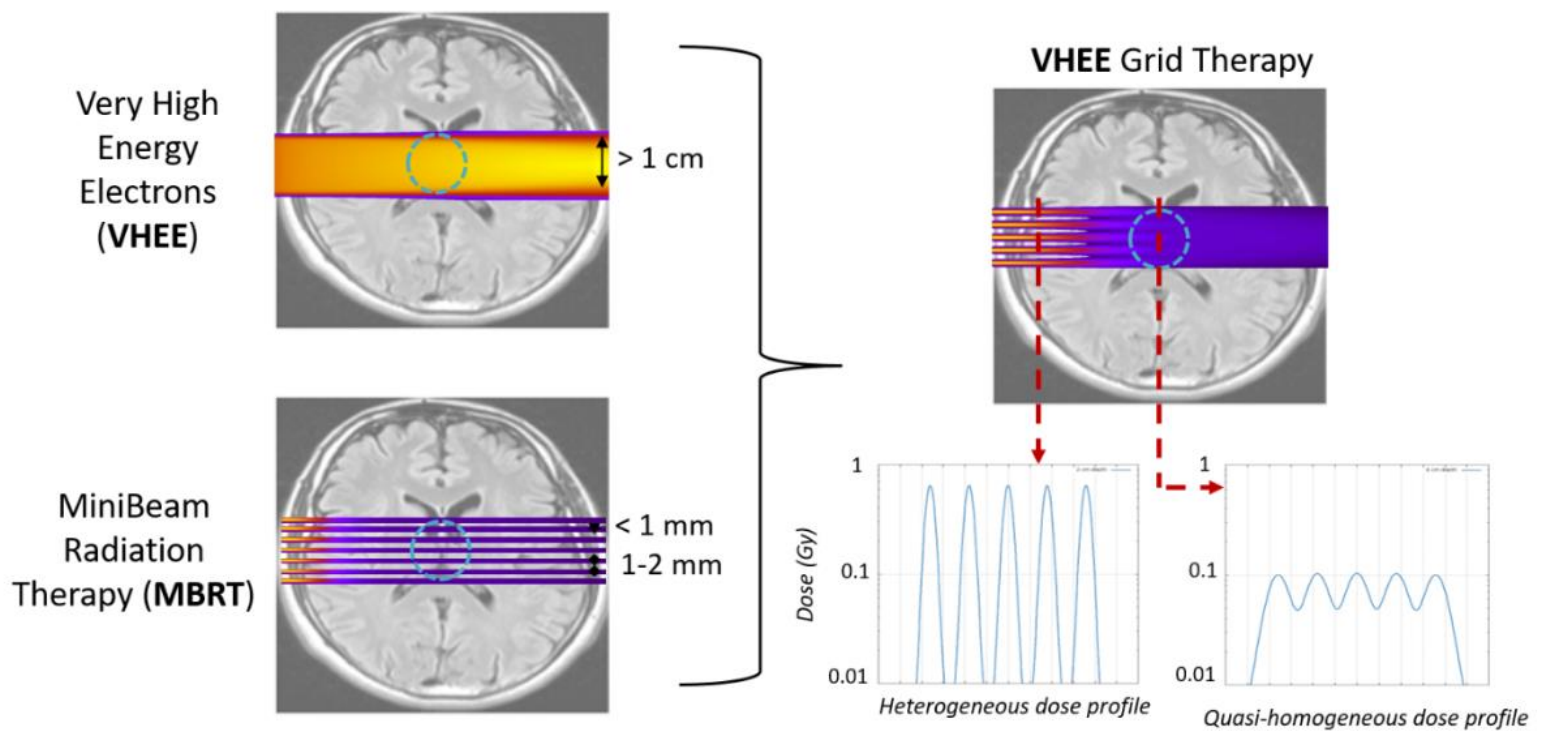

Figure 2: Principle of the spatial fractionation of dose and VHEE GRID therapy with two examples of peak and valley dose profiles.

The combination of VHEE with the benefits of GRID therapy has been recently proposed [8]. One advantage of using charged beams is that sub-millimetric beam sizes can be obtained with pure magnetic collimation, avoiding the degradation of the grid pattern on the cumulated dose due to secondary particles coming from a collimator. Varying physical parameters such as beam energies, grid sizes and grid spacing, it is possible to favor tumor exposure by delivering a quasi-homogeneous dose in the tumor, or to enhance the normal tissue tolerance by enlarging the fractionated dose area. Figure 2 illustrates the basic principle of VHEE grid therapy, with examples of peaks and valleys dose profiles in depth. The first objective of the radiobiology project at PRAE is to implement this fully innovative approach on the PRAE deviated beamline in order to evaluate its therapeutic benefit in sparing normal tissues. The high-performance of the beam is a key point of the success of this investigation which requires sub-millimetric beam sizes, small beam divergence, high energies, high stability and reproducibility. First theoretical studies demonstrated the 
feasibility of such an implementation at PRAE [9]. Preliminary experimental dosimetry measurements will validate the technique and motivate preclinical studies.

Beyond the demonstration that a compact, performant and cost-effective machine can be constructed, the PRAE facility intends in a second phase to provide beam for biological experiments (in vitro and in vivo) to deepen the knowledge on VHEE therapy.

\section{The ProRad experiment}

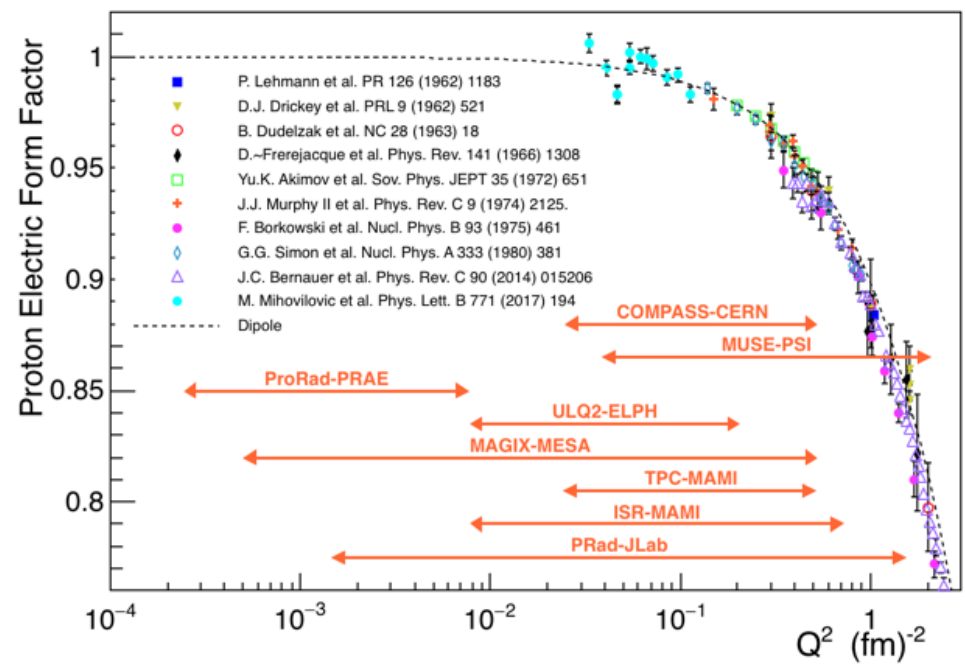

$$
\begin{aligned}
& \text { Figure 3: The } \\
& \mathrm{Q}^{2} \text { coverage of } \\
& \text { the worldwide } \\
& \text { low- } \mathrm{Q}^{2} \\
& \text { experimental } \\
& \text { effort } \\
& \text { compared to } \\
& \text { existing data } \\
& \text { on the proton } \\
& \text { electric form } \\
& \text { factor. }
\end{aligned}
$$

The ProRad (Proton Radius) experiment aims at providing new high accuracy data in an unexplored ultra-low four-momentum transfer $\left(Q^{2}\right)$ domain, to help understand the still present proton charge radius puzzle $[2,10]$. After about a decade and several new measurements, the difference between muonic hydrogen spectroscopy [11] and elastic electron scattering [12] measurements of the charge radius of the proton $\left(R_{p}\right)$ is not yet understood. An unprecedented low- $Q^{2}$ experimental effort (Fig. 3 ) is developing worldwide to assess $R_{p}$ as measured from the scattering technique. Part of the problem comes from the fact that the subatomic method does not provide a direct measurement, but accesses $R_{p}$ through the zero-extrapolation of the $\mathrm{Q}^{2}$-slope of the electric form factor of the proton. Forthcoming data at low- $Q^{2}$ are expected to provide a better measurement of $R_{p}$ by firmly controlling the extrapolation procedure.

As many experiments in this $Q^{2}$-range, ProRad is facing several challenges to be able to reach significant precision. ProRad is designed to provide $0.1 \%$ accurate measurements of the ratio of the cross sections between elastic and Møller electron scatterings. The setup follows simple and robust considerations for particle identification, position, and energy measurements in a non-magnetic environment at energies well below the pion production threshold. The reaction chamber hosts a windowless target made of a $15 \mu \mathrm{m}$ diameter solid hydrogen jet [13], effectively concretizing a pure and nearly point-like target. A $2 \mathrm{~m}$ long vacuum vessel is closed by an end-cap which supports the detector elements. They consist of 28 elementary cells organized symmetrically around the beam and located at different scattering angles. The elementary cell is composed of two layers of thin scintillator strips followed by a cylindrical BGO crystal. The scintillator hodoscope acts all at once as a neutral particle discriminator, a charged particle tagger, and a position detector which defines the electron scattering angle. The same BGO crystal simultaneously measures elastic and Møller electrons which are distinguished at each scattering angle by their different energies. 
The dominant sources of ProRad systematic uncertainties originate from the knowledge of the absolute incident electron beam energy $\left(E_{0}\right)$ and the scattered electron angles. To achieve the necessary precision on $E_{0}\left(5 \times 10^{-4}\right)$, the ProRad beamline will feature a magnetic dipolar deviation where the beam deviation angle and the field integral along the beam trajectory will be accurately measured. Thanks to the PRAE beam properties and the quasipoint-like nature of the target, the positioning of the hodoscope is the main source of angle systematics. With a position accuracy better than $100 \mu \mathrm{m}$ ProRad will achieve $0.05 \mathrm{mrad}$ systematical error on the scattering angle. Altogether, ProRad is an experimental challenge at the precision frontier.

\section{Acknowledgements}

We would like to thank Y. Prezado (IMNC, Orsay, France), S. Barsuk, D. Douillet, A. FausGolfe, V. Puill (LAL, Orsay, France), and P. Duchesne, C. Le Galliard, B. Genolini, E. Voutier (IPN, Orsay, France) for their deep involvement in the PRAE facility project as scientific and technical coordinators. The PRAE platform is supported by the French Ile-de-France region within the framework of the SESAME 2017 program, the LabEx Physique des 2 Infinis et des Origines (ANR-10-LABX-0038) within the framework of Investissements d'Avenir (ANR-11IDEX-0003-01), the LIA IDEATE, and the French Centre National de la Recherche Scientifique.

\section{References}

1. (PRAE Collaboration) D. Marchand et al. EPJ Web of Conf. 138 (2017) 010102.

2. J.C. Bernauer, R. Pohl, Sci. Am. 310 (2014) 32.

3. (PRAE Collaboration) S. Barsuk et al. IPAC 2017, JACoW (2017) THPVA079.

4. C. DesRosiers, V. Moskvin, F. Bielajew, L. Papiez, Phys. Med. Biol. 45 (2000) 1781.

5. E. Schüler et al. Med. Phys. 44 (2017) 2544.

6. M. Mohiuddin et al. Int. J. Radiat. Oncol. Biol. Phys. 45 (1999) 721.

7. Y. Prezado et al. J. Synchrotron Radiat. 19 (2012) 60.

8. I. Martínez-Rovira, G. Fois, Y. Prezado, Med. Phys. 42 (2015) 685.

9. R. Delorme, A. Hrybok, A. Faus-Golfe, Y. Prezado, Radiother. Oncol. 127 (2018) S1214.

10. A. Marsman et al. Phys. Rev. A. 98 (2018) 012509.

11. R. Pohl et al., Nature 466 (2010) 213.

12. (A1 Collaboration) J.C. Bernauer et al. Phys. Rev. C 90 (2014) 015206.

13. R. A. Costa Fraga et al. Rev. Sci. Inst. 83 (2012) 025102. 